\title{
Depressão Puerperal, no âmbito da Saúde Pública
}

\author{
Francislene Lopes Menezes*, Neida Luiza Kaspary Pellenz ${ }^{* *}$, \\ Suzinara Soares de Lima***, Fernanda Sarturi****
}

RESUMO: A depressão pós-parto (DPP) é uma doença expressiva ocorrência, aproximadamente 10 a $20 \%$ das mulheres sofrem desse transtorno psiquiátrico, sendo considerado um importante problema de saúde pública. Esta doença caracteriza-se por humor depressivo, cansaço, desânimo, perda de prazer, com início, em geral, nas primeiras quatro semanas após o parto. Assim, esta pesquisa tem por objetivos, analisar os procedimentos necessários para diminuir os índices nacionais de DPP, pesquisar a capacitação sobre a DPP para equipes nas Unidades Básicas de Saúde e caracterizar as implicações da DPP na interação mãe-bebê. Trata-se de pesquisa bibliográfica de abordagem qualitativa. Os procedimentos para melhorar a saúde materna e impossibilitar mortes evitáveis é um grande desafio. Embora, a DDP continue sendo um objeto de estudo ainda incipiente existem instrumentos que auxiliam para sua identificação precoce. A capacitação de profissionais da saúde deve ser intensificada, pois as implicações na interação mãe-bebê são graves.

Descritores: Pessoal de saúde, Saúde da Mulher, Depressão pós-parto.

\section{Postpartum depression under public health}

Abstract: Postpartum depression is a significant disease; approximately from 10 to $20 \%$ of the women suffer from this psychiatric disorder which is considered an important public health problem. This disease is characterized by depressed mood, fatigue, depression, loss of pleasure, which usually starts within the first four weeks after child-birth. This study aims to analyze the required or necessary procedures to lower the national indices of postpartum depression (DPP), to research training on the DPP for health teams in the Basic Health units and characterize the implications of the DPP in the interaction between mother and baby or mother-infant interaction. This is a qualitative approach on bibliographical research. The procedures for improving maternal health and preclude preventable deaths are great challenge; eventhoug the DDP is A still inceptive object of study, there are tools that help on its early identification. The capacitation of health workers should be intensified, because the implications for mother-infant interaction are severe.

Descriptors: Health personnel, Women's Health, Postpartum Depression.

*Enfermeira. Mestranda do Programa de Pós-Graduação em Enfermagem na Universidade Federal de Santa Maria (UFSM), Santa Maria, RS, Brasil.

${ }^{* *}$ Enfermeira. Mestre em Enfermagem pela Universidade Federal de Santa Catarina (UFSC), Florianópolis, SC, Brasil.

${ }^{* * *}$ Enfermeira. Doutora em Enfermagem pela Escola de Enfermagem Anna Néry - Universidade Federal do Rio de Janeiro, Rio de Janeiro, RJ, Brasil.

${ }^{* * * * E n f e r m e i r a . ~ M e s t r e ~ e m ~ E n f e r m a g e m ~ p e l a ~ U n i v e r s i d a d e ~ F e d e r a l ~ d e ~ S a n t a ~ M a r i a ~(U F S M), ~ S a n t a ~ M a r i a, ~ R S, ~ B r a s i l . ~}$ 


\section{Introdução}

A sociedade contemporânea busca diariamente o idealismo e o perfeccionismo. As mulheres, desde a infância são preparadas para serem mães e constituírem família. Entretanto, o que muitas vezes parece algo intrínseco ao ser mulher, pode se constituir em um falso idealismo, pois nem sempre, ser mãe ocorre como foi construído no imaginário de cada mulher.

Tornar-se mãe acarreta várias mudanças físicas e emocionais para a mulher, as quais se intensificam ao longo da gestação e após o parto. Tais mudanças podem ocasionar doenças que se desencadeiam no puerpério, entre elas, a depressão, a qual possui características semelhantes aos demais transtornos do humor, exceto pelos pensamentos e sentimentos de culpa, devido à incapacidade para atuar como mãe, incluindo também, a possibilidade de ocorrerem sintomas psicóticos. Quando há presença de delírios, eles frequentemente envolvem fantasias associadas ao recém-nascido, como por exemplo, que 0 bebê está possuído pelo demônio, ter poderes especiais ou ter pela frente um destino terrível'.

Para ser diagnosticada, a depressão puerperal inclui pelo menos cinco dos sintomas listados a seguir, os quais devem ser manifestados quase todos os dias: uma grande tristeza de natureza prolongada, baixa auto estima, crise de choro, fadiga, desmotivação, ansiedade, distúrbios do sono e/ou do apetite, irritabilidade, sensação de incapacidade para cuidar do filho ou desinteresse por ele, lapsos de memória e ideias obsessivas ou supervalorizadas de suicídio.

A depressão pós-parto apresenta uma incidência de aproximadamente $10 \%$ a $20 \%$ de casos. Contudo, somente $50 \%$ dos casos são diagnosticados na clínica diária e menos de $25 \%$ das puérperas acometidas pela doença têm acesso ao tratamento 2 .

Vários são os fatores de risco associados à etiologia da depressão pós-parto (DPP). Entre eles, encontram-se o histórico familiar de depressão; mulheres que sofrem de tensão pré-menstrual (TPM); problemas de infertilidade; dificuldades na gestação; gestantes submetidas à cesariana, cujo parto, não é considerado o mais adequado para enfrentar a demanda emocional do puerpério, pois, como toda cirurgia, tende a debilitar a paciente no pós-operatório, baixando suas resistências e interferindo na qualidade do seu humor; as primigestas; as mulheres em situação econômica precária; as mães solteiras; as mulheres que perderam pessoas importantes no período gestacional; aquelas que vivem em desarmonia conjugal ou que se casaram em função da gravidez; a ansiedade materna; um suporte pós-natal frági $\left.\right|^{3}$. Ainda, dentre esses fatores, existem as situações relacionadas ao bebê, entre elas, as intercorrências neonatais, como a prematuridade e as malformações congênitas.

Atualmente, a depressão puerperal é considerada um importante problema de saúde pública em nosso país, afetando tanto a saúde da mãe, quanto o desenvolvimento normal do bebê. A doença acontece, principalmente, a partir das primeiras quatro semanas após 0 parto, atingindo sua intensidade máxima, nos seis primeiros meses depois do nascimento do bebê4 ${ }^{4}$. Os transtornos psiquiátricos, no período puerperal, são reconhecidos pela instalação dos sintomas e desenvolvimento dos mesmos. Geralmente são classificados em três níveis: melancolia pós-parto, depressão puerperal e psicose puerperal. 
Embora, havendo as classificações mencionadas no parágrafo acima, o diagnóstico é difícil de ser realizado, pois o quadro clínico muda sua apresentação e a intensidade dos sintomas, à medida que a doença evolui. As puérperas, muitas vezes, negligenciam os sinais que se manifestam e a família, na maioria das vezes, acaba atribuindo o "cansaço e a tristeza" às mudanças da rotina doméstica e aos afazeres com o bebê.

Não existem parâmetros fisiológicos para avaliar as manifestações clínicas da depressão puerperal, mas escalas de avaliação são utilizadas para medir e caracterizar os sintomas, mesmo não podendo ser consideradas como critério de diagnóstico. Dentre as escalas de autoavaliação, encontramos a Escala de Depressão Pós-Parto de Edimburgo (EPDS), uma das mais utilizadas para realizar o rastreamento de sintomas depressivos que se manifestam após o parto, tendo sido traduzida para 24 idiomas, com estudos de validação na maioria dos países, incluindo o Brasili, ${ }^{51}$. As escalas são autoaplicáveis e de fácil utilização por profissionais não médicos e sem especialização em saúde mental. Segundo eles as escalas auxiliariam para alertar clínicos, obstetras e pediatras em relação às mulheres que possivelmente, precisariam de avaliação mais profunda e de tratamento.

O interesse em desenvolver este estudo, direcionado à Saúde da Mulher, surgiu a partir dos resultados dos dados obtidos no Trabalho de Conclusão do Curso de Graduação de Enfermagem, intitulado: "Frequência da depressão puerperal e o conhecimento das enfermeiras da maternidade do HU/FURG acerca dessa doença" da Universidade Federal do Rio Grande (FURG), onde foi possível perceber que existe uma lacuna quanto à detecção e atendimento relativo a este importante problema de saúde pública ${ }^{7}$. Os estudos evidenciam que não existe uma proposta de cuidado específico, realizada pela equipe de saúde, a fim de prevenir ou prestar a assistência adequada o mais precocemente possível desse transtorno mental.

Tendo em vista que o conhecimento da DPP é indispensável aos profissionais da saúde que atuam na área obstétrica, pediátrica e da família, em função de prestarem cuidados diretos às puérperas e seus familiares, é imprescindível que saibam identificar a instabilidade e/ou labilidade emocional destas e direcionem as ações de cuidado, no sentido de ajudá-las a enfrentarem e superarem as dificuldades encontradas neste momento de transição do ciclo vital.

Este estudo tem o propósito de analisar os procedimentos que podem melhorar os índices de puérperas acometidas pela depressão pós-parto no contexto da atenção básica, pesquisar de capacitação sobre a DPP para equipes nas Unidades Básicas de Saúde e caracterizar as implicações da DPP na interação mãe-bebê.

\section{Metodologia}

Este estudo trata de uma revisão bibliográfica, de caráter qualitativo, iniciando com uma leitura preliminar utilizando-se de um trabalho de conclusão Curso de Enfermagem da autora, livros e artigos da área de Enfermagem Obstétrica e Ginecológica quanto de Psiquiatria. Posteriormente, foi realizada uma busca eletrônica utilizando como palavras-chaves depressão pós-parto, saúde da mulher e profissionais de saúde. Foi selecionado um total de doze artigos, utilizados para o desenvolvimento do estudo.

Saúde (Santa Maria), v.38, n.1, p. 21-30, 2012. Menezes, F.L., et al. 
No trabalho de conclusão ${ }^{7}$, foi realizada uma coleta de dados com 53 puérperas e cinco enfermeiras da maternidade. Os dados foram coletados em duas etapas distintas. Na primeira ocorreu a aplicação do questionário baseado na escala EPDS (Edinburgh Post-natal Depression Scale), um instrumento que avalia a saúde da puérperas, especificamente, no que se refere à detecção precoce do risco de estar desenvolvendo sintomas depressivos. $\mathrm{Na}$ segunda etapa, foi utilizada uma entrevista semiestruturada com as enfermeiras, o qual buscou caracterizá-las e identificar os conhecimentos delas a respeito da escala EPDS, os saberes formais que possuem acerca da depressão puerperal e suas intervenções em relação à detecção precoce da doença. A frequência de risco encontrada no estudo foi de $11 \%$, demonstrando a importância de os profissionais da saúde em realizarem a deteç̧ão precoce da depressão pós-parto, bem como, receberem capacitação para a área da saúde mental, pois é necessário para promoverem a saúde da mulher, do bebê e da família. As enfermeiras consideraram importante receberem conhecimentos relativos a como aplicarem a escala, bem como, um aprofundamento dos primeiros cuidados que devem realizar com a família das puérperas consideradas como de risco para desencadearem a DPP.

Após a leitura e reflexão dos resultados obtidos no referido trabalho de conclusão, este artigo apresenta uma discussão dos resultados obtidos, os quais foram subdivididos em novas categorias como: saúde da mulher no Brasil, pré-natal e diagnóstico da depressão puerperal, possíveis implicações da DPP na interação mãe-bebê, a gestão pública em saúde e as ações dos profissionais voltadas à saúde da mulher.

\section{Resultados e discussão}

\section{Saúde da mulher no Brasil}

O Ministério da Saúde preconiza diversas políticas e ações, visando promover a saúde nas diferentes porções da população brasileira, sendo que todas são fundamentadas nos princípios do Sistema Único de Saúde (SUS). A saúde da mulher possui a Política Nacional de Atenção Integral a qual possui o programa de "Assistência Integral à Saúde da Mulher: Bases de Ação Programática" (PAISM). O PAISM propõe que a assistência seja prestada em todas as fases da vida, clínica, ginecológica, reprodução (planejamento reprodutivo, gestação, parto e puerpério) como nos casos de doenças crônicas ou agudas, reconhecendo a assistência como cuidado médico e de toda a equipe de saúde, priorizando práticas educativas, que compreendem a capacidade crítica e a autonomia das mulheres ${ }^{8}$.

O Ministério da Saúde ainda ressalta que a Norma Operacional da Assistência à Saúde (NOAS), editada pela Portaria do dia 27 de fevereiro de $2002{ }^{9}$, tem como finalidade ampliar as responsabilidades dos Municípios na Atenção Básica, criando mecanismos de fortalecimento da gestão do SUS, atualizando os critérios de habilitação para os Estados e Municípios $^{8}$. Sendo que, dentre estas responsabilidades da Atenção Básica à Saúde a serem executadas estão às ações da Saúde da Mulher, voltadas a prevenção, planejamento familiar

Saúde (Santa Maria), v.38, n.1, p. 21-30,

2012. Depressão puerperal no âmbito da saúde pública $24 \mid$

ISSN 2236-5834 e pré-natal.

Em junho de 2000, o Ministério da Saúde instituiu o Programa de Humanização no PréNatal e Nascimento (PHPN) ${ }^{10}$, para assegurar acesso e qualidade do acompanhamento ao pré-natal, da assistência ao parto, pós-parto e neonatal ${ }^{8}$. Logo, foi criado o Sistema de 
Acompanhamento do Programa de Humanização no Pré-Natal e Nascimento (SIS Pré-Natal), um programa informatizado que subsidia Municípios, Estados e o Ministério da Saúde com informações fundamentais para o planejamento, acompanhamento e avaliação das ações desenvolvidas, através PHPN.

O programa SIS Pré-Natal tem por objetivo primordial reduzir as altas taxas de morbimortalidade materna, perinatal e neonatal no país. Baseia-se no direito inalienável da cidadania. Portanto, direito ao acesso, por parte das gestantes e dos recém-nascidos, à assistência à saúde nos períodos pré-natal, parto, puerpério e neonatal, tanto na gestação de baixo como de alto risco, por meio da organização adequada dos serviços de saúde, assegurando a integralidade da assistência e com investimentos e custeios necessários ${ }^{11}$.

O Pacto Nacional pela Redução da Mortalidade Materna e Neonatal, lançado em março de 2004, traz, como uma de suas ações estratégicas, saúde da mulher portadora de transtornos mentais, visando promover o diagnóstico oportuno e tratamento às mulheres com quadro de depressão puerperal ${ }^{8}$.

A melhoria das condições de saúde da mulher depende do êxito das ações de saúde desenvolvidas nas unidades básicas, de responsabilidade de todos e executadas por uma equipe multiprofissional composta por: agente comunitário de saúde (ACS), auxiliar de enfermagem, enfermeiro, nutricionista, médico, dentista e assistente social, que atuam por meio do atendimento individual ou em grupo e adequados às necessidades da mulher, da família e da comunidade. As unidades básicas concedem estabelecer um relacionamento mais próximo dos profissionais de saúde com a população.

As mulheres representam $50,77 \%$ da população brasileira e são as principais usuárias do SUS. Elas frequentam os serviços de saúde para o seu próprio atendimento, mas também acompanhando parentes ou não, além de por vezes assumir o papel de cuidadoras de membros da família, vizinhos, ou comunidade ${ }^{12}$. Esses são alguns aspectos que os profissionais de saúde devem estar atentos, em especial o enfermeiro, pois atua na prevenção primária, cuidado individual e coletivo, tendo como ponto central a família como um todo. Podendo realizar diagnóstico de gravidez, consulta de enfermagem no pré-natal, intercalada à médica, visita domiciliar a gestantes e puérperas, entre outras ações.

\section{O pré-natal e o diagnóstico da depressão puerperal}

O pré-natal configura-se como consultas programadas e realizadas pelas gestantes com os integrantes da equipe de saúde a fim de monitorar a evolução da gravidez, a preparação adequada para o parto, 0 aleitamento materno e os cuidados com o bebê. Tais consultas também objetivam a deteç̧ão de doenças maternas, a prevenção, o diagnóstico precoce e 0 tratamento das complicações da gravidez, além de exercer a vigilância do crescimento e da vitalidade fetal. Logo, o pré-natal refere-se aos cuidados médicos e de enfermagem prestados à mulher grávida entre a concepção e 0 trabalho de parto ${ }^{13}$.

O manual técnico "Pré-natal e Puerpério" do Ministério da Saúde coloca que

...muitos dos sintomas físicos manifestos mascaram problemáticas subjacentes. Por isso, em vez de fazer uma série de rápidas perguntas, específicas e fechadas, é importante encorajar a mulher a falar de si. Essa abordagem é chamada de "entrevista centrada na pessoa". Saber ouvir é tão importante quanto saber o que dizer, pois essa habilidade pode ser crucial para a elaboração de um diagnóstico correto ${ }^{13, p .35}$.

Saúde (Santa Maria), v.38, n.1, p. 21-30,
2012.
Menezes, F.L., et al.
ISSN 2236-5843 | 25


O diagnóstico da depressão puerperal é difícil, muitas vezes ele passa despercebido pelos profissionais da saúde. Após o parto, é comum a preocupação com aspectos físicos da mãe e do bebê, e o psicológico deixa de ser investigado.

Após a alta hospitalar, a puérpera faz geralmente uma consulta com o obstetra, geralmente o único atendimento de saúde recebido. Nessa consulta essencialmente faz-se 0 controle da involução das modificações gravídicas e o início da contracepção deixando-se, erroneamente, de lado as alterações de humor, distúrbios emocionais e da sexualidade ${ }^{14}$.

A detecção precoce da DPP poderia ser realizada através da prevenção primária e secundária de saúde, nas maternidades e acompanhamento sistemático das mães nos períodos do pré-natal, perinatal e pós-parto, tanto nos hospitais, como unidades básicas de saúde. Já existem escalas que descrevem o rastreamento da depressão pós-parto, a Edinburgh Post-Natal Depression Scale (EPDS) e a Postpartum Depression Screening Scale (PDSS), ambas traduzidas para o português e validadas no Brasil.

Um estudo realizado por enfermeiras, com o objetivo de identificar a prevalência e os fatores de risco para depressão puerperal e sua associação com transtornos mentais, nas puérperas atendidas em duas unidades do Programa de Saúde da Família do município de São Paulo, utilizaram como um dos instrumentos, a EPDS. Destacando que, devido à dificuldade das puérperas terem acesso aos serviços de saúde mental, existe a necessidade de um instrumento adequado para detectar a DPP precocemente, a fim de que, posteriormente, elas sejam encaminhadas para as consultas psiquiátricas. Desse modo, a aplicação da EPDS seria uma estratégia importante para rastrear a DPP ${ }^{15}$

O reconhecimento da depressão puerperal precocemente evita problemas na relação conjugal, pois mulheres com DPP podem ser incapazes de satisfazerem suas próprias necessidades e as do seu companheiro; não conseguem perceber os gestos de amor e carinho demonstrados por ele e sentem-se como se fossem um "peso" na vida da família. 0 cônjuge, na maioria das vezes, percebe tal situação de maneira confusa e pode apresentar sentimentos de estresse, negação e raiva. Outras manifestações encontradas nos homens é a sensação de serem negligentes e se sentirem culpados ${ }^{16}$. 0 afastamento e a interrupção da atenção maternal ao bebê podem ocasionar problemas para o desenvolvimento saudável.

\section{Possíveis implicações da DPP na interação mãe-bebê}

A depressão puerperal pode ser um ponto crucial no desenvolvimento e crescimento saudável de uma criança. A tendência dos bebês é tornarem-se vulneráveis às consequências da depressão materna, pois dependem totalmente da qualidade dos cuidados e da responsabilidade da mãe.

É no puerpério que ocorre uma interação cada vez mais estreita entre a mãe e o seu bebê, por isso, nesse período é essencial que a mãe esteja bem emocional e fisicamente, a fim de estabelecer o envolvimento afetivo com seu filho ${ }^{17}$. A extensão do impacto da depressão materna para o filho depende de vários fatores, entre eles, a idade da criança; 0 temperamento da criança; a cronicidade do episódio depressivo materno e do próprio estilo

ISSN 2236-5834 interativo da mãe deprimida, o qual poderá ser apático ou intrusivo. Os resultados, geralmente são sérios para a saúde da mulher e do bebê, pois os danos causados no estabelecimento do vínculo entre esta díade podem persistir pela vida inteira, caso a puérpera e sua família não recebam o tratamento adequado, na DPP ${ }^{18}$. 
Um estudo ${ }^{17}$ realizado em Ribeirão Preto, para avaliar e comparar os perfis de interação mãe-bebê, em mulheres com depressão pós-parto e mães que não apresentavam essa condição, mostrou que a presença de sintomas depressivos no pós-parto estão diretamente relacionados aos danos que ocorrem na interação da mãe com o seu bebê.

No período da adolescência, a mãe continua exercendo um importante papel no desenvolvimento das habilidades emocionais, biológicas, sociais e no caráter do filho. Um estudo ${ }^{19}$ com 240 mães e seus filhos adolescentes, os quais receberam acompanhamento anual e forneceram informações acerca do impacto que a depressão materna, detectou que dentre os adolescentes, cujas mães apresentaram episódios depressivos, nas fases iniciais do desenvolvimento destes, posteriormente, aumentou o número de usuários de drogas ilícitas, iniciação precoce da atividade sexual e houve maiores taxas de evasão escolar. Os filhos de mães depressivas são considerados indivíduos que apresentam alto risco para apresentarem problemas, ao longo do desenvolvimento, necessitando desta forma, receber maior atenção no micro espaço familiar e escolar.

\section{A gestão pública em saúde e as ações dos profissionais voltadas à saúde da mulher}

Os gestores e trabalhadores da saúde pública devem sustentar suas decisões considerando que assistência prestada à mulher deve ser focada no período gravídicopuerperal, incluindo o parto e nascimento; analisando aspectos físicos e psicológicos. Procurando sempre melhorar o conhecimento sobre assuntos voltados a saúde da mulher portadora de transtornos mentais, logo, aperfeiçoando as decisões relacionadas a esse assunto.

O Pacto Nacional pela Redução da Mortalidade Materna e Neonatal do Ministério da Saúde tem como ação qualificar os profissionais da rede SUS para o atendimento da mulher portadora de transtornos mentais, promovendo 0 acolhimento especializado nos serviços de atenção Obstétrica e Neonatal. A rede básica tem que investir em seus profissionais da saúde, pois existe uma fragilidade na capacitação desses para atender problemas psicológicos.

A equipe de enfermagem é muito visada, pois é ela que recebe os pacientes nas unidades, realiza intervenções no pré-natal e fica maior parte nas unidades básicas de saúde. A identificação do problema várias vezes é percebida por esses profissionais.

O modelo assistencial proposto pela Estratégia da Saúde da Família (ESF) é fundamentado na promoção à saúde e na prevenção de doenças e agravos, buscando atender 0 indivíduo em seu contexto familiar e comunitário. Assim, o enfermeiro da ESF deve ter o conhecimento acerca da DPP para atuar no acolhimento e direcionamento adequado da gestante durante um pré-natal contínuo, humanizado e integral, numa lógica de prevenção deste transtorno mental. No decorrer do pré-natal este desenvolve ações preventivas, voltadas não somente à saúde da gestante, mas à saúde integral da mulher. Conhecendo 0 contexto sócio familiar da gestante, identificando fatores de risco para a DPP e realizando intervenções de apoio emocional ainda no ensejo da consulta de pré-natal ${ }^{20}$.

O estudo ${ }^{21}$ ressalta a importância da escala EPDS como instrumento para triagem da DPP, pois é de fácil aplicação, rápido, baixo custo e possibilidade de aplicação por qualquer profissional de saúde. Salienta-se que seu uso no SUS poderia repercutir positivamente com aumento significativo na taxa de reconhecimento, diagnóstico, e tratamento da depressão pós-parto.

Saúde (Santa Maria), v.38, n.1, p. 21-30, 2012.

Menezes, F.L., et al. 


\section{Considerações finais}

O estudo demonstrou que os desafios são grandes, mas que podem ser superados. As escalas de autoavaliação que existem nesta área podem facilitar o rastreamento de sintomas depressivos que se manifestam após o parto e, também, permitir a difusão de cursos de capacitação para as equipes de saúde na sistematização do cuidado.

O Brasil possui vários programas voltados para a Saúde da Mulher, os quais necessitam otimizar sua implementação. Os gestores necessitam priorizar em suas atividades a saúde mental, uma especificidade muitas vezes esquecida. Os profissionais da saúde percebem esta lacuna, mas não recebem os incentivos para colocar em prática seus conhecimentos.

Vários estudos ${ }^{18,17,19}$ abordam que a interação mãe-filho é fortemente afetada, trazendo consequências até mesmo na fase de adolescência. Portanto, torna-se cada vez mais visível a necessidade de mobilizar ações preventivas e educativas referentes a esta temática.

O transtorno mental apontado com maior frequência no período pós-natal é a depressão, fazendo-se necessário que os profissionais da saúde estejam sensibilizados e capacitados para realizarem ações eficazes quanto à promoção da saúde da mulher, família e da criança. Os profissionais de saúde devem se unir, não delegando estas ações somente aos psiquiatras e psicólogos, mas sim, procurar construir uma rede de referência que contribua com a ampliação das intervenções primária e secundária frente à doença.

\section{Referências}

1. American Psychiatry Association - DSM IV. Manual Diagnóstico e Estatistico de Transtornos Mentais. $4^{\mathrm{a}}$. ed, Porto Alegre: Artes Medicas; 2002.

2. Ruschi GEC, Sun SY, Mattar R, Filho AC, Zandonade E, Lima VJ. Aspectos epidemiológicos da depressão pós-parto em amostra brasileira. Rev psiquiatr Rio Gd Sul [periódico online] 2007 Set-Dez [capturado 2011 Mar 1]; 29(3):274-280. Disponivel em: http://www.scielo.br/pdf/rprs/v29n3/v29n3a06.pdf

3. laconelli V. Depressão pós-parto, psicose pós-parto e tristeza materna. Rev Pediatr Mod [periódico online] 2005 Jul-Ago 41[capturado 2011 fev 2]; (4):210-213, Disponível em:

http://www.cibersaude.com.br/revistas.asp?fase=r003\&id_materia=3063

4. Moraes IGS. et al. Prevalência da depressão pós-parto e fatores associados. Rev Saude Publica [periódico online] 2006 Jan-Fev [capturado 2011 fev 23]; 40(1): 65-70. Disponível em: http://www.scielo.br/pdf/rsp/v40n1/27117.pdf

5. Santos MFS, Martins FC, Pasquali L. Escalas de auto-avaliação de depressão pós-parto: estudo no Brasil. Rev Psiquiatr Clin [periódico online] 1999 Mar-Abr [capturado 2011 fev 1];26(2):32-40. Disponível em: http://www.hcnet.usp.br/ipq/revista/vol26/n2/artigo(90).htm

6. Camacho RS. et al. Transtornos psiquiátricos na gestação e no puerpério: classificação, diagnóstico e tratamento. Rev Psiquiatr Clin [periódico online] 2006 Mar-Abr [capturado 2011 fev 4]; 33(2):92-102. Disponivel em: http://www.honet.usp.br/ipq/revista/vol33/n2/pdf/92.pdf

7. Menezes FL. Frequência da depressão puerperal e o conhecimento das enfermeiras da maternidade do

Saúde (Santa Maria), v.38, n.1, p. 21-30,

2012.

Depressão puerperal no âmbito da saúde pública 28 HU/FURG acerca dessa doença. Rio Grande: [s.n], 2009. 62p. Monografia (Trabalho de Conclusão de Curso) - Universidade Federal do Rio Grande. Escola de Enfermagem, 2009.

8. Ministério da Saúde (BR). Pacto nacional pela redução da mortalidade materna e neonatal. [online]. 2011 [acessado 2011 Fev 26]. Disponível em: http://portal.saude.gov.br/portal/saude/odm_saude/visualizar_texto.cfm?idtxt=35197 
9. Ministério da Saúde (BR). Legislação. [online]. 2002 [acessado 2011 Fev 27]. Disponivel em: http://bvsms.saude.gov.br/bvs/saudelegis/gm/2002/prt0373_27_02_2002.html

10. Ministério da Saúde (BR). Secretaria de Políticas de Saúde. A implantação da Unidade Básica da Saúde da Família. Ministério da Saúde, Brasilia: 2000.

11. Ministério da Saúde (BR). Sistema Pré-natal. O Sisprenatal. Ministério da Saúde; 2008. [acessado em: 02 mar 2011]. Disponivel em: http://sisprenatal.datasus.gov.br/SISPRENATAL/index.php?area=01

12. Ministério da Saúde (BR). Secretaria de Atenção à Saúde. Departamento de Ações Programáticas Estratégicas. Política nacional de atenção integral à saúde da mulher: princípios e diretrizes. Ministério da Saúde, Brasilia: 2007.

13. Ministério da Saúde (BR). Secretaria de Atenção a Saúde. Departamento de Ações Programáticas Estratégicas. Área Técnica de Saúde da Mulher. Pré- natal e Puerpério: atenção qualificada e humanizada manual técnico. Ministério da Saúde, Brasília: 2006.

14. Stefanello J, Nakano MAS, Gomes FA. Crenças e tabus relacionados ao cuidado no pós-parto: 0 significado para um grupo de mulheres. Acta paul. Enfermagem [periódico online] 2008 [capturado $2011 \mathrm{Mar}$ 3]; 21(2):275-28. Disponivel em: http://www.scielo.br/pdf/ape/v21n2/pt_a07v21n2.pdf

15. Cruz EBS, Simoes GL, Cury AF. Rastreamento da depressão pós-parto em mulheres atendidas pelo Programa de Saúde da Família. Rev. Bras. Ginecol. Obst. [periódico online] 2005 Abr [capturado 2011 Mar 8]; 27(4)181-188. Disponivel em: http://www.scielo.br/pdf/rbgo/v27n4/a04v27n4.pdf

16. Lowdermilk DL, Perry SE, Bobak, IM. O cuidado em enfermagem materna. 5a. ed. Porto Alegre: Artmed;2002.

17. Ramos SHAS, Furtado EF. Depressão puerperal e interação mãe-bebê: um estudo piloto. Rev. Psicol. Pesq. [periódico online] 2007 Jan-Jun [capturado 2011 Mar 1];1(1): 20-28. Disponível em: http://www.uff.br/psicologiaempesquisa/files/2009/12/v1n1004.pdf

18. Frizzo GB, Piccinini CA. Interação mãe-bebê em contexto de depressão materna: Aspectos teóricos e empíricos. Rev. Psicol. estud. [periódico online] 2005 Jan-Abr [capturado 2011 Fev 23]; 10(1): 47-55. Disponível em: http://www.scielo.br/pdf/pe/v10n1/v10n1a06.pdf

19. Depressão materna afeta comportamento dos filhos. Jornal Tudo Bem [online] 2007 Fev 15; Bem Estar, [capturado 2011 Fev 24]. Disponivel em: http://gambare.uol.com.br/2007/02/15/depressao-materna-afetacomportamento-dos-filhos/

20. Valença CN, Germano RM. Prevenindo a Depressão Puerperal na Estratégia Saúde da Família: Ações do Enfermeiro no Pré-Natal. Rev. Rene [periódico online] 2010 Abr-Jun [capturado 2011 Fev 23]; 11 (2): 129139. Disponível em: http://www.revistarene.ufc.br/vol11n2_pdf/a15v11n2.pdf

21. Figueira P, Corrêa H, Malloy-Diniz L, Romano-Silva MA. Escala de Depressão Pós-natal de Edimburgo para triagem no sistema público de saúde. Rev Saude Publica [periódico online] 2009 Ago [capturado 2011 Mar 3]; 43 Suppl.1:79-84.Disponível em: http://www.scielo.br/scielo.php?pid=S003489102009000800012\&script=sci_arttext

\section{Francislene Lopes Menezes}

Endereço para correspondência - Rua Prof. Heitor da Graça Fernandes, nº310 - Apto 301.

Camobi, CEP 97105-170, Santa Maria/RS.

E-mail: francislenelm@yahoo.com.br

Currículo Lattes: http://lattes.cnpq.br/3309506991914979

Recebido em 02 de outubro de 2011.

2012.

Menezes, F.L., et al. 
Saúde (Santa Maria), v.38, n.1, p. 21-30,

2012. Depressão puerperal no âmbito da saúde pública 30 ISSN 2236-5834 